\title{
Spectral properties of milling and machined surface
}

\author{
Gabor STEPAN ${ }^{1, a^{*}}$, Mate TOTH ${ }^{1, b}$, Daniel BACHRATHY ${ }^{1, c}$ and \\ Suri GANERIWALA ${ }^{2, d}$
}

${ }^{1}$ Department of Applied Mechanics, Budapest University of Technology and Economics, Muegyetem rkp 3, Budapest H-1111 HUNGARY

${ }^{2}$ SpectraQuest Inc., 8227 Hermitage Road, Richmond VA 23228 USA

astepan@mm.bme.hu, btoth_m@mm.bme.hu, cbachrathy@mm.bme.hu, dsuri@spectraquest.com

${ }^{*}$ corresponding author

Keywords: chatter, milling, spectrum, surface quality

\begin{abstract}
Machine tool vibrations cause uncomfortable noise, may damage the edges of cutting tools or certain parts of machine tools, but most importantly, they always have negative effect on the quality of the machined surface of workpieces. These vibrations are especially intricate in case of milling processes where complex tool geometries are used, like helical, serrated, non-uniform pitch angles, and so on. During the milling process, the arising vibrations include free, forced, selfexcited, and even parametrically forced vibrations together with their different combinations. Regarding surface quality, the most harmful is the self-excited one called chatter, which is related to the regenerative effect of the cutting process. Its relation to machined surface quality is demonstrated in an industrial case study. The modelling and the corresponding cutting stability are presented in case of a helical tool applied for milling with large axial immersions. The extremely rich spectrum of the measured vibration signals are analyzed by means of model-based predictions, and the results are compared with the spectral properties of the corresponding machined surfaces. The conclusions open the way for new kinds of chatter identification.
\end{abstract}

\section{Introduction}

Stability charts are widely used for predicting and avoiding machine tool chatter (see, for example [1-3]). However, the well-defined theoretical stability boundaries can rarely be identified perfectly in practice. The distinction of stable and unstable cutting processes is still a challenging task in the vicinity of marginally stable parameter combinations due to parameter uncertainties especially regarding structural and process damping as well as nonlinearities in the cutting force characteristics [4].

The rich spectrum of vibration signals corresponding to chatter becomes even more complex in the presence or machine tool runout, when the geometric axis of the tool does not coincide perfectly with its axis of rotation [5]. Runout can never be eliminated perfectly, and its presence can be detected both in stable and unstable cutting processes.

The most relevant frequency components of the rich spectrum can be traced on the machined surface as well, which provides a way to identify machine tool chatter based on the surface finish itself (see, for example [6,7]). In this study, the two-dimensional Fourier transformation [8] is introduced as an effective method to capture intricate details of the machined surface and to distinguish stable and unstable cutting processes this way.

\section{Mechanical model of milling}

A single degree-of-freedom (DoF) model of milling is presented in Fig. 1. The tool is assumed to be flexible with a single relevant vibration mode in the feed direction, and the workpiece is considered to be rigid. The corresponding equation of motion reads 


$$
\ddot{x}(t)+2 \zeta \omega_{\mathrm{n}} \dot{x}(t)+\omega_{\mathrm{n}}^{2} x(t)=\frac{1}{m} F_{x}(t),
$$

where $\omega_{\mathrm{n}}$ is the angular natural frequency, $\zeta$ is the damping ratio, $m$ denotes the modal mass, while $F_{x}$ is the $x$ component of the resultant cutting force acting on the tool. Figure 1 shows an upmilling operation with spindle speed $\Omega$ given in rpm.

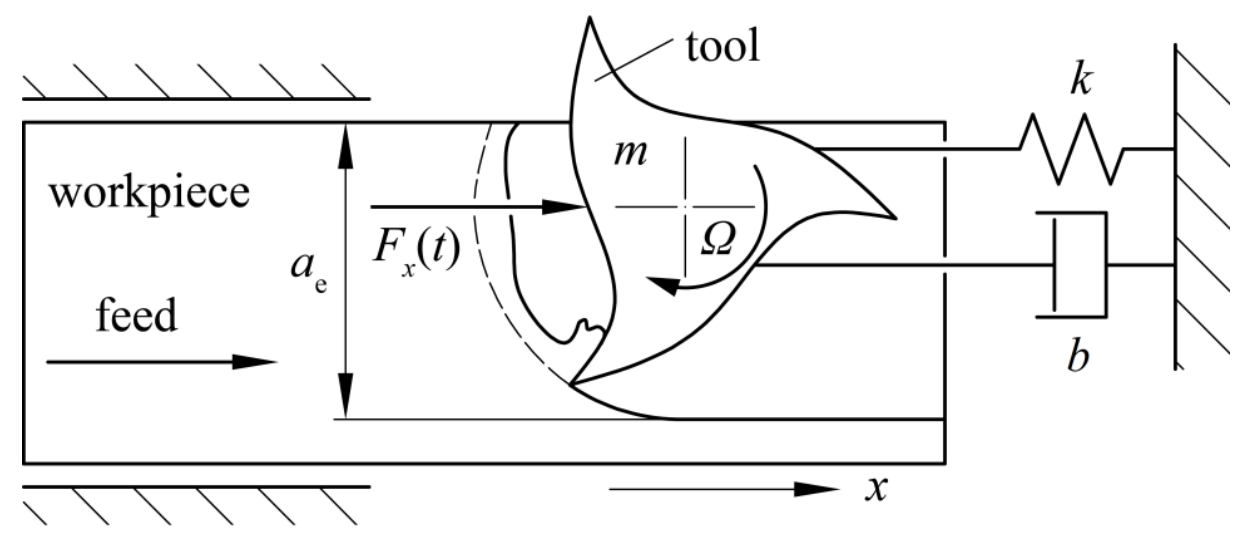

Fig. 1: Mechanical model of milling

A helical milling tool is considered with number $Z$ of teeth and helix pitch $l_{\mathrm{p}}$. In case of helical tools, the forces acting on the cutting edges change along the axial direction $z$ of the tool. The tangential and radial cutting force components acting on tooth $j$ assume the forms

$$
\begin{aligned}
& F_{j, \mathrm{t}}(t)=\int_{0}^{a_{\mathrm{p}}} g_{j}(t, z) K_{\mathrm{t}} h_{j}^{q}(t, z) \mathrm{d} z, \\
& F_{j, \mathrm{r}}(t)=\int_{0}^{a_{\mathrm{p}}} g_{j}(t, z) K_{\mathrm{r}} h_{j}^{q}(t, z) \mathrm{d} z,
\end{aligned}
$$

where $z$ is the coordinate along the axial direction of the tool, $K_{\mathrm{t}}$ and $K_{\mathrm{r}}$ are the tangential and radial cutting force coefficients [2], respectively, $h_{j}$ is the actual chip thickness cut by tooth $j, q$ is the cutting force exponent, and $g_{j}$ is a screen function determining whether tooth $j$ is in $\left(g_{j}=1\right)$ or out $\left(g_{j}=0\right)$ of the cut [7]. The actual cutting forces are calculated by means of the integration along the $z$ axis from zero to the axial immersion $a_{\mathrm{p}}$. In the argument of these integrals, the chip thickness cut by tooth $j$ can be calculated as

$$
h_{j}(t, z)=\left(f_{\mathrm{z}}+x(t-\tau)-x(t)\right) \sin \varphi_{j}(t, z),
$$

where $f_{\mathrm{z}}$ is the constant feed per tooth. The surface regeneration effect [2] is described by $x(t-\tau)$ and $x(t)$, which denote the delayed and actual positions of the tool, respectively, where $\tau=60 /(Z \Omega)$ is the time delay or tooth pass time, and $\varphi_{j}$ presents the actual angular position of tooth $j$.

When all these are substituted back to the equation of motion (1), the general solution has a periodic (or forced) component $x_{\mathrm{p}}(t)$ and its variation $\xi(t)$ can be interpreted as the perturbation of the stationary milling process. When this general solution $x(t)=x_{\mathrm{p}}(t)+\xi(t)$ is substituted to Eq. (1), the linearized equation of motion around the periodic solution assumes the form

$$
\ddot{\xi}(t)+2 \zeta \omega_{\mathrm{n}} \dot{\xi}(t)+\left(\omega_{\mathrm{n}}^{2}+G\left(t, a_{\mathrm{p}}\right)\right) \xi(t)=G\left(t, a_{\mathrm{p}}\right) \xi(t-\tau),
$$

where $G$ is a particular directional factor [7] that depends on the axial immersion $a_{\mathrm{p}}$ linearly, and it is a periodic function of the time $t$. 
The stability of the zero solution of Eq. (5) corresponds to stable stationary milling process, while its instability refers to the appearance of chatter. The stability properties of Eq. (5) are checked by means of the semi-discretization method [9]. The stability boundaries are located where the modulus of the critical characteristic multiplier $\mu_{1}$ is equal to one. The characteristic multipliers are calculated from Eq. (5) based on Floquet Theory (see details in [9]).

The chatter frequencies $\omega_{\mathrm{c}}$ can be found by utilizing the expression $\mu_{1}=\cos \left(\omega_{\mathrm{c}} T\right)+\mathrm{i} \sin \left(\omega_{\mathrm{c}} T\right)$, where the time period $T$ is equal to the tooth pass period (or time delay), that is, $T=\tau$ if the runout is negligible. For this case, the qualitative diagram of Fig. 2 shows the distinction between forced and self-excited vibration frequencies above the parameter domain of the spindle speed.

Note that in the presence of runout, the time period is equal to the spindle period, that is, $T=60 / \Omega=Z \tau$. In this case, the harmonics of the forced vibration frequency and the chatter frequencies are scaled down by the number $Z$ of the cutting edges, which leads to an even richer spectrum in the frequency domain.

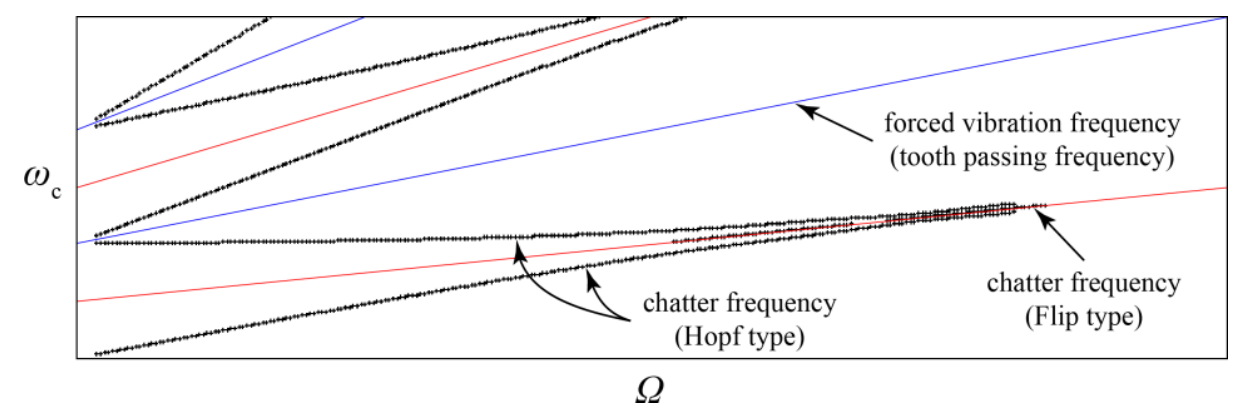

Fig. 2: Forced and self-excited vibration frequencies

\section{Measurement}

Milling experiments were carried out in the workshop of SpectraQuest Inc. The tool presented in the left panel of Fig. 3 was a high speed steel Niagara Cutter 33905 end mill with a diameter of $25.4 \mathrm{~mm}$, helix angle of $37^{\circ}$, and $Z=3$ cutting teeth. The corresponding helix pitch could be calculated as $35.3 \mathrm{~mm}$.

A triaxial sensor assembly was mounted on the tool holder and two additional accelerometers were attached to the base of the milling machine as shown in the right panel of Fig. 3. The labels $\mathrm{H}$, $\mathrm{V}$ and $\mathrm{A}$ stand for horizontal, vertical and axial directions of the tool, respectively. The modal parameters were determined by standard impulse hammer tests [10], which resulted $\omega_{\mathrm{n}}=3977 \mathrm{rad} / \mathrm{s}$, $\zeta=0.053$ and $m=0.231 \mathrm{~kg}$ that were considered cylindrically symmetric in the $\mathrm{H}$ and $\mathrm{V}$ directions.

The applied feed rate was kept at $6.35 \mathrm{~mm} / \mathrm{s}$ during all the milling experiments discussed below for unstable and stable cases.

\section{Unstable milling}

The measurement results presented in the panels of Fig. 4 correspond to down-milling with spindle speed $\Omega=4500 \mathrm{rpm}$, axial immersion $a_{\mathrm{p}}=25.4 \mathrm{~mm}$ and radial immersion $a_{\mathrm{e}}=1.6 \mathrm{~mm}$. The acceleration signals were captured by the sensor labeled 'Tool_V' in Fig. 3, which points in the feed direction. The frequency resolution of the recorded data was $0.39 \mathrm{~Hz}$.

The stability chart in panel d) of Fig. 4 and the corresponding theoretical frequency diagram in panel c) were determined based on the previously detailed mechanical model of milling for the estimated parameters $K_{\mathrm{t}}=33 \cdot 10^{6} \mathrm{~N} / \mathrm{m}^{q+1}, K_{\mathrm{r}}=11 \cdot 10^{6} \mathrm{~N} / \mathrm{m}^{q+1}$ with the standard exponent $q=0.75$ (see, for example, [2,11]).

Due to the fixture of the tool in the tool holder by means of a worm-screw, the presence of runout was relevant in the experiments, which is indicated by the orange dots in the measured spectrum in panel a) of Fig. 4. The quality of the machined surface finish of the workpiece in panel c) of Fig. 4 corresponds to the unstable milling process. 

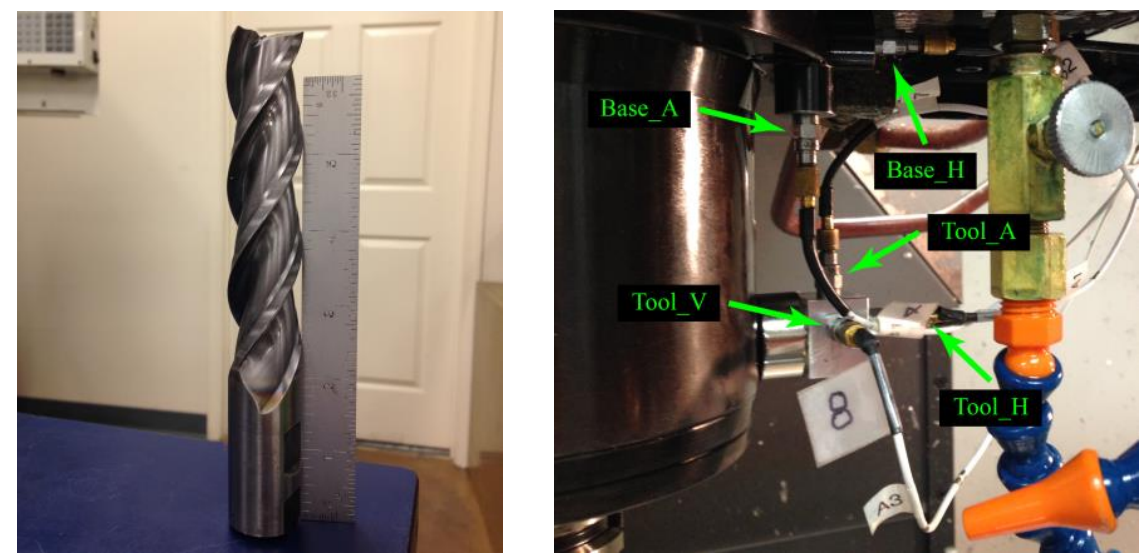

Fig. 3: Three-fluted end mill and measurement setup

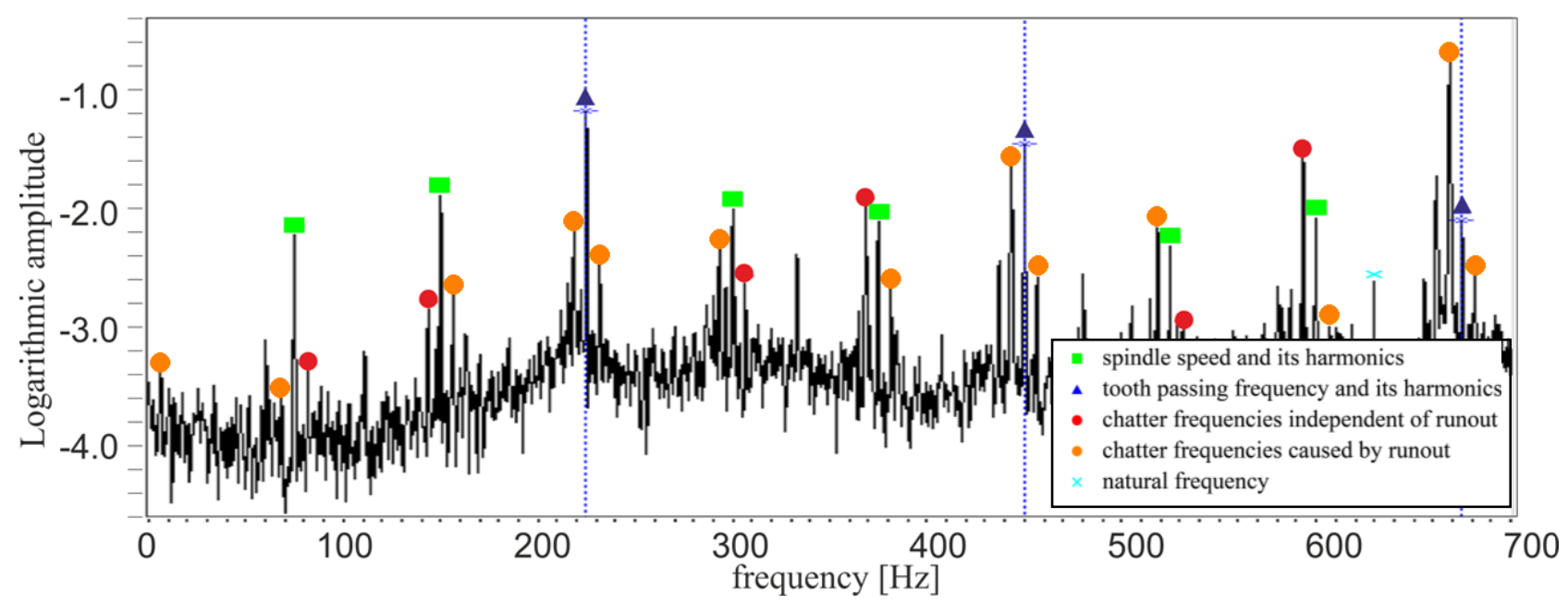

Surface finish:
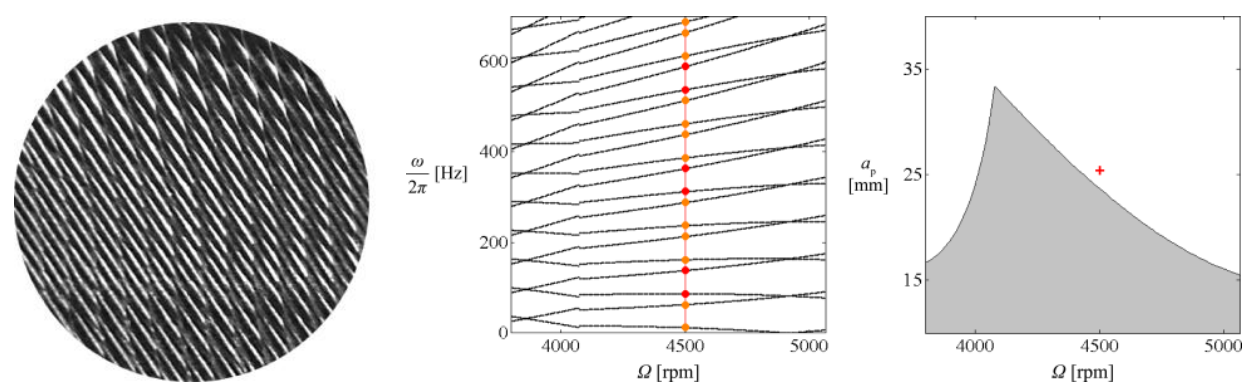

Fig. 4: Theoretical and measurement results for unstable down-milling with the indication of runout: measured spectrum with dots for the theoretically estimated frequencies in panel a); machined surface in panel b); predicted vibration frequencies in panel c); and calculated stability chart with the parameter point (red cross) of the measurement in panel d)

Fig. 4 shows that the presence of runout introduces additional chatter frequencies into the spectrum of the measured vibration signal. In a runout-free case, the chatter frequencies are located and correspond only to the tooth passing frequency $1 / \tau$. However, in the presence of runout, the principal period of the system is shifted from the tooth passing period to the spindle rotation period [5]. Consequently, additional chatter frequencies appear corresponding to the spindle rotation frequency, while the chatter frequencies related to the tooth passing frequency remain unchanged in the spectrum.

Table 1 summarizes the comparison of the theoretical calculations and the measurement results. It can be seen that even the single DoF model of milling described above predicts the arising chatter frequencies with great accuracy. 


\begin{tabular}{|c|c|c||c|c|c|}
\hline Measurement & \multicolumn{2}{|c||}{ Theoretical model } & Measurement & \multicolumn{2}{c|}{ Theoretical model } \\
\hline $\begin{array}{c}\text { frequency } \\
{[\mathrm{Hz}]}\end{array}$ & $\begin{array}{c}\text { frequency } \\
{[\mathrm{Hz}]}\end{array}$ & $\begin{array}{c}\text { error } \\
{[\%]}\end{array}$ & $\begin{array}{c}\text { frequency } \\
{[\mathrm{Hz}]}\end{array}$ & $\begin{array}{c}\text { frequency } \\
{[\mathrm{Hz}]}\end{array}$ & $\begin{array}{c}\text { error } \\
{[\%]}\end{array}$ \\
\hline 6.64 & 11.94 & 79.79 & 368.36 & 363.09 & -1.43 \\
\hline 68.36 & 63.07 & -7.74 & 381.64 & 387.02 & 1.41 \\
\hline 81.64 & 86.97 & 6.53 & 443.36 & 438.10 & -1.19 \\
\hline 143.36 & 138.06 & -3.70 & 456.64 & 462.03 & 1.18 \\
\hline 156.64 & 161.98 & 3.41 & 518.36 & 513.11 & -1.01 \\
\hline 218.36 & 213.07 & -2.42 & 531.64 & 537.04 & 1.02 \\
\hline 231.64 & 237.00 & 2.31 & 593.36 & 588.12 & -0.88 \\
\hline 293.36 & 288.08 & -1.80 & 606.64 & 612.05 & 0.89 \\
\hline 306.64 & 312.01 & 1.75 & 668.36 & 663.38 & -0.74 \\
\hline
\end{tabular}

Table 1: Comparison of the experimental and theoretical vibration frequency components

\section{Stable milling}

The experimental results presented in Fig. 5 correspond to the same parameters as those of Fig. 4, except for the spindle speed, which is reduced to $\Omega=1000 \mathrm{rpm}$.

The spectral resolution of the recorded data remained $0.39 \mathrm{~Hz}$, and the displayed frequency range relative to the applied spindle speed was equal to that of the case of $\Omega=4500 \mathrm{rpm}$; this is the reason why the spectrum appears to be less refined in panel a) of Fig. 5.

The theoretical chatter frequencies are marked by red dashed lines in panel a) of Fig. 5. Since these are only approximations, the real chatter frequencies are to be looked in the vicinity of each theoretically calculated frequency value. In a few cases, small peaks can be detected near some of these lines, but in general, the experimental spectrum shown in panel a) of Fig. 5 is free from chatter frequencies.

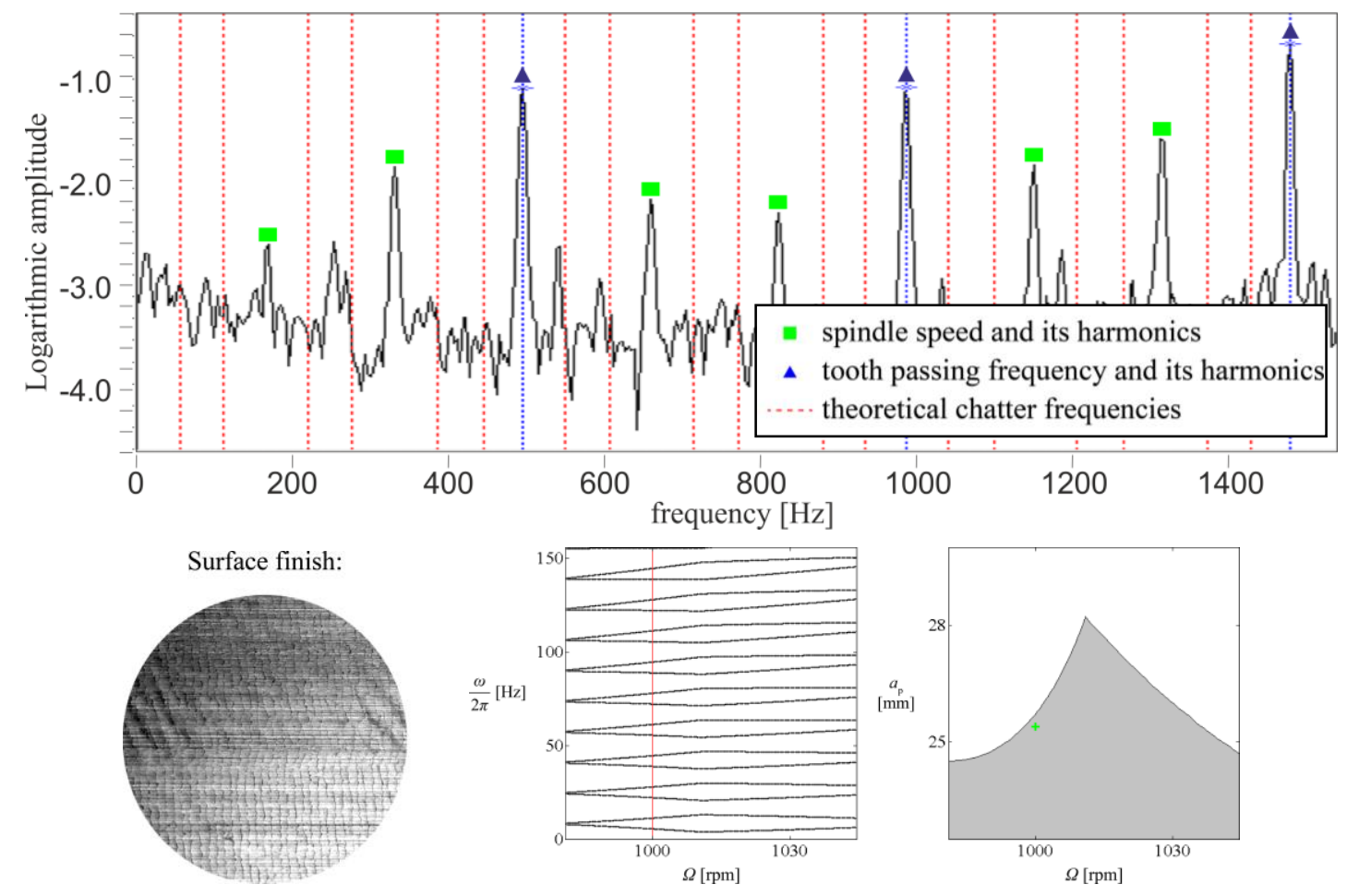

Fig. 5: Stable down-milling: spectrum in panel a), machined surface in panel b), theoretically predicted vibration frequencies in panel c) and stability chart in panel d). 
The large peaks corresponding to the combined presence of chatter and runout are completely missing for $\Omega=1000 \mathrm{rpm}$. In the case of $\Omega=4500 \mathrm{rpm}$, these peaks are so significant that they can be easily seen even by using a linear amplitude scale. For $\Omega=1000 \mathrm{rpm}$, the linear amplitude scale barely shows any frequencies other than the spindle speed and its harmonics which clearly include the tooth passing frequency and also its harmonics.

\section{Spectral properties of the machined surface}

The two-dimensional Fourier transformation (2DFFT) is an effective way to investigate the spectral properties of an image. The method can be used for decomposing an image into a series of two-dimensional sinusoidal waves of different amplitudes, frequencies and directions. The amplitude spectrum of an image is represented in the plane of two wave numbers (or spatial frequencies) which are perpendicular to each other. The visual representation of this concept is shown in Fig. 6.

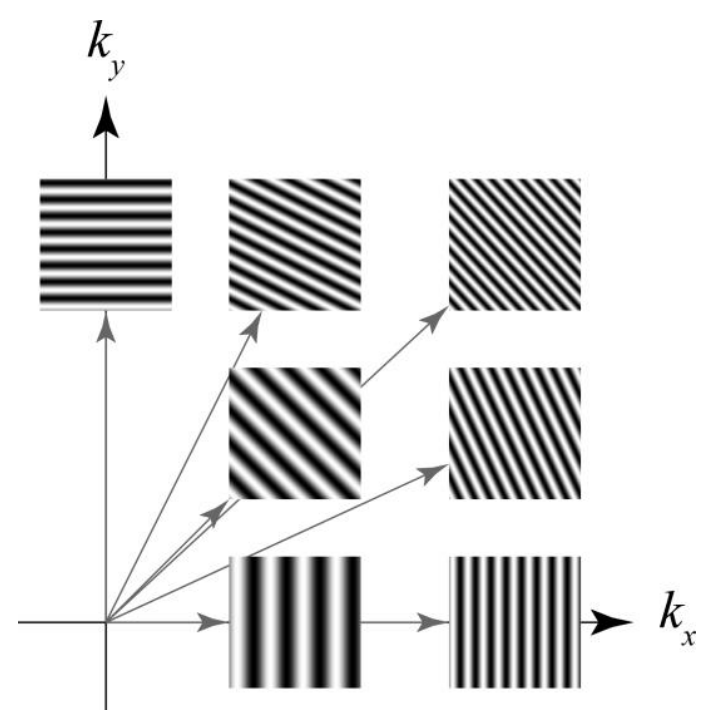

Fig. 6: Constituent waves of the two-dimensional Fourier transformation

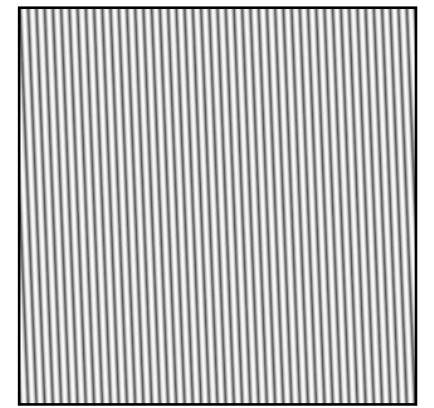

a) No vibration

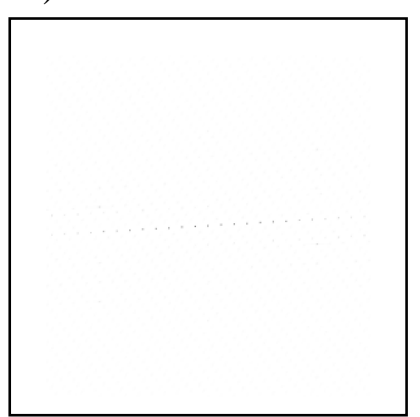

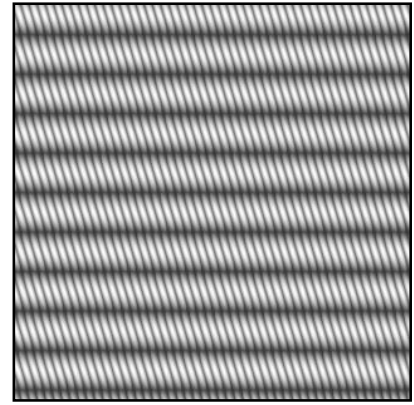

b) Forced vibration

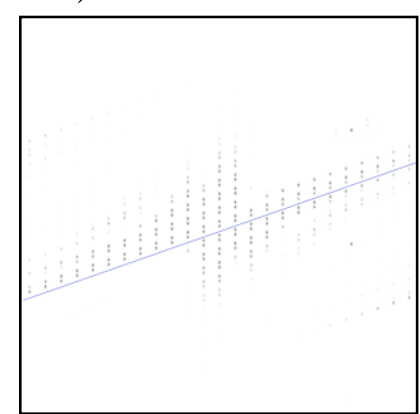

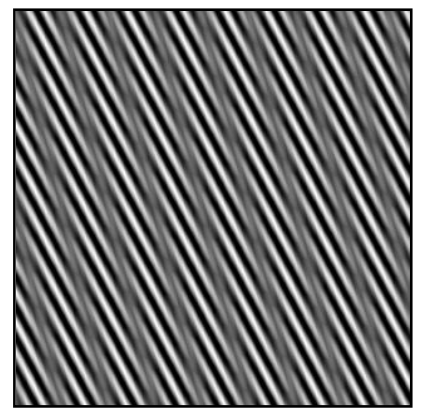

c) Chatter vibration

Fig. 7: Numerical simulation of the machined surface corresponding to no vibration a), forced $b$ ) and self-excited c) vibrations, and the corresponding 2DFFTs 
Figure 6 presents the spatial frequencies $k_{x}$ and $k_{y}$ with dimension $1 / \mathrm{m}$ and the directions of propagation as position vectors. The larger the magnitudes of these vectors are, the higher the frequencies of the corresponding sinusoidal waves are. Therefore, the low-frequency spectral components are located around the origin of the coordinate system spanned by $k_{x}$ and $k_{y}$, while the high-frequency components are found further away from the origin.

Figures 7 and 8 show numerically generated surface segments taken from [6] and pictures of the machined surfaces described in the above experiments, respectively. The corresponding 2DFFTs are also presented below the pictures. Panels a), b) and c) of Fig. 7 present cases of milling processes with no vibration, forced vibration and chatter vibration, respectively.

Figures $7 \mathrm{a}$ and $8 \mathrm{a}$ show that in the case of stable milling operations the vibrations are negligible, and the only significant spectral property of the machined surface is related to the marks belonging to the feed per tooth. For helical tools, the inclination angle of these marks is defined by the helix pitch and the feed per tooth.

Even in case of stable milling operations, forced vibrations may result in a rough surface finish as presented in Fig. $7 \mathrm{~b}$ and $8 \mathrm{~b}$. Due to the nature of the arising vibrations, the inclination angle of the surface marks (indicated by a blue straight line) is clearly identical to the helix angle of the milling tool.

In the case of the (self-excited) chatter vibrations, the helix angle of the milling tool (blue line in Fig. 8c) is still very prominent in the spectrum, however, an additional pattern along the indicated red line is formed which is related to the chatter frequency of the cutting process.

We can state, that the numerically generated 2DFFT spectra and the measurement based 2DFFT spectra of the machined surfaces show the same pattern, apart from some noise existing in the experiments.

\section{Summary}

In this paper, the stability of milling processes was studied in the presence of relevant runout effect. The model-based theoretical results and the corresponding measurements showed that the presence of runout causes additional vibration frequencies that are not present in a runout-free machining operation.

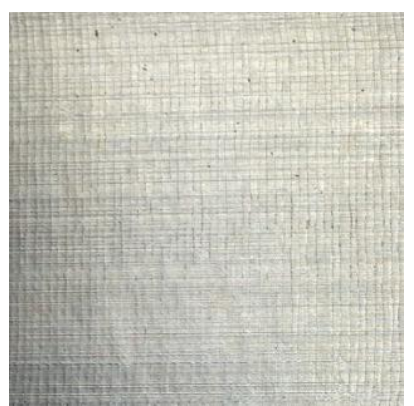

a) Negligible vibration

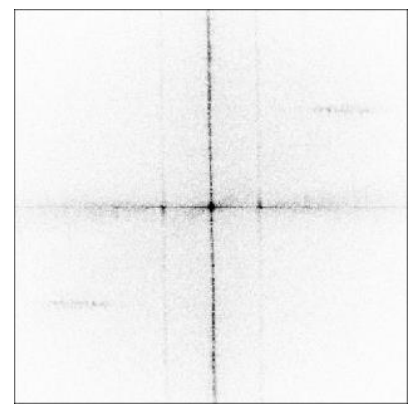

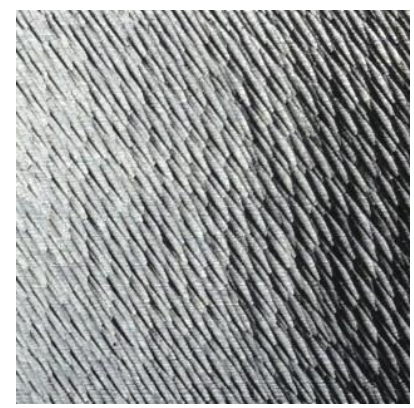

b) Forced vibration

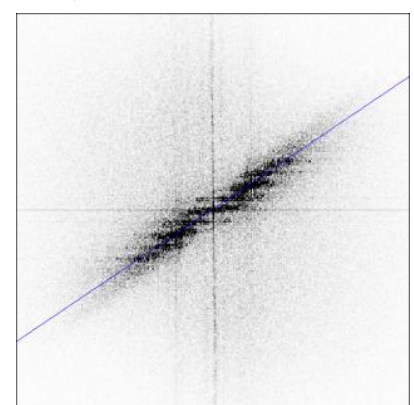

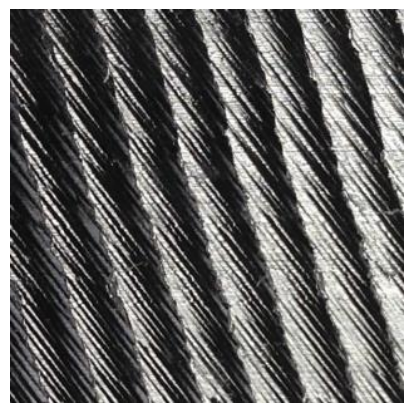

c) Chatter vibration

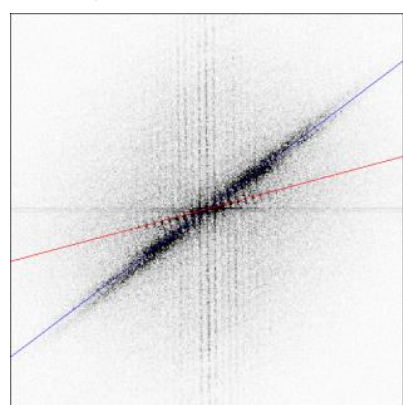

Fig. 8: Surface finishes corresponding to negligible a), forced b) and self-excited c) vibrations together with their corresponding 2DFFTs 
The application of the two-dimensional Fourier transformation was introduced to extract detailed information for the milling process from the machined surface finish. The efficiency of the method was demonstrated by providing an easy-to-use method that makes clear distinction between milling processes of different stability properties.

\section{Acknowledgment}

The research leading to these results has received funding from the European Research Council under the European Union's Seventh Framework Programme (FP7/2007-2013) ERC Advanced grant agreement No340889 and form the Hungarian National Science Foundation under grant no. K101714.

\section{References}

[1] E. Budak and Y. Altintas, Analytical Prediction of Chatter Stability in Milling - Part I: General Formulation. Journal of Dynamic Systems Measurement and Control 120 (1998) 22-30.

[2] Y. Altintas, Manufacturing Automation: Metal Cutting Mechanics, Machine Tool Vibrations, and CNC Design, Cambridge University Press, Cambridge, 2000.

[3] G. Stepan, Retarded Dynamical Systems, Longman, Harlow, 1989.

[4] G. Stepan, Z. Dombovari and J. Munoa J Identification of Cutting Force Characteristics Based on Chatter Experiments. CIRP Annals - Manufacturing Technology 60 (2011) 113-116.

[5] T. Insperger T, B.P. Mann, T. Surmann and G. Stepan G, On the chatter frequencies of milling processes with runout, International Journal of Machine Tools and Manufacture, 48 (2008) 10811089.

[6] B. Denkena, M. Krueger, D. Bachrathy and G. Stepan, Model based reconstruction of milled surface topography from measured cutting forces. International Journal of Machine Tools \& Manufacture 54-55 (2012) 25-33.

[7] D. Bachrathy, T. Insperger and G. Stepan, Surface properties of the machined workpiece for helical mills, Machining Science and Technology 13 (2009) 227-245.

[8] D.E. Dudgeon and R.M. Mersereau, Multidimensional Digital Signal Processing, Prentice Hall, New York, 1983.

[9] T. Insperger and G. Stepan, Semi-discretization Method for Time-Delay Systems, Springer, New York, 2011.

[10] D. J. Ewins, Modal Testing - Theory, Practice \& Application, $2^{\text {nd }}$ Ed., Baldock, Hertfordshire, 2000.

[11] E. Budak and Y. Altintas, Prediction of milling force coefficients from orthogonal cutting data. Journal of Manufacturing Science and Engineering, 118 (1996) 216-225. 